DOI 10. $18307 / 2021.0220$

(C) 2021 by Journal of Lake Sciences

\title{
青藏高原拉昂错热力分层和混合层深度变化特征观测”
}

\author{
苏荣明珠 ${ }^{1,2}$,马伟强 ${ }^{1,3 * *}$,马耀明 ${ }^{1,2,3}$, 谢志鹏 ${ }^{1}$, 王宾宾 ${ }^{1}$, 胡 伟 $^{1,2}$, 刘景时 ${ }^{1}$ \\ (1: 中国科学院青藏高原研究所, 北京 100101) \\ (2: 中国科学院大学, 北京 100049) \\ (3:中国科学院青藏高原地球科学卓越创新中心, 北京 100101)
}

\begin{abstract}
摘 要: 湖泊热力结构不仅影响湖泊内部生态环境, 而且与区域气象和气候系统相互影响,但目前对湖泊垂直温度的观 测研究仍非常匮乏. 本研究基于青藏高原拉昂错连续的湖温和气象观测, 分析了小时尺度和日尺度热力分层规律和混合 层深度的变化特征. 结果表明: 拉昂错为冷多次完全混合型湖泊; 湖表温度 8 月达到最大值, 湖面敞水区和沿岸的湖表温 度季节震荡相同, 但存在一定的空间差异; 与空气温度相比, 湖表温度变幅小, 降温更慢, 峰值滞后约 1 个月. 同时发现, 拉昂错混合层深度变化较为规律, 辐射和风速是影响湖泊混合层深度的主要外界因子. 探明真实的高原湖泊分层规律, 有利于提高湖泊模拟能力, 为优化湖泊参数化方案提供参考.
\end{abstract}

关键词: 湖温;热力分层;混合层深度;青藏高原; 拉昂错

\section{Investigation of thermal stratification and mixed layer depth in La'ang Co in the Tibetan Plateau *}

Su Rongmingzhu ${ }^{1,2}$, Ma Weiqiang ${ }^{1,3 * *}$, Ma Yaoming ${ }^{1,2,3}$, Xie Zhipeng ${ }^{1}$, Wang Binbin ${ }^{1}$, Hu Wei ${ }^{1,2} \&$ Liu Jingshi ${ }^{1}$

(1: Institute of Tibetan Plateau Research, Chinese Academy of Sciences, Beijing 100101, P.R.China)

(2: University of Chinese Academy of Sciences, Beijing 100049, P.R.China)

(3: The Center for Excellence in Tibetan Plateau Earth Sciences (CETES) of the Chinese Academy of Sciences, Beijing 100101, P.R.China)

\begin{abstract}
Lake mixing regimes not only provide ecosystem services to the lacustrine status but also interact with the regional climate. However, field observational data on the vertical water temperature of lakes is scarce on the Tibetan Plateau, which inhabits a clear understanding of the actual thermal structures of lakes in high cold regions. Based on the water temperature profiles and meteorological data of La'ang Co in the Tibetan Plateau, variations of thermal stratification and mixed layer depth in the hourly and daily scale were investigated. La'ang Co is a cold polymictic lake, and the lake surface temperature peaks in August. The water temperature in the lake centre and along the shoreline exist spactial variability, although both exhibited similar seasonal fluctuations. The surface temperature decreases slowly and stably, which lags behind the air temperature about a month. Furthermore, the variation of the mixed depths in La'ang Co is formed, and solar radiation and wind are key external factors in regulating the mixed layer depths. A better understanding of the mechanism of the lake stratification on the Tibetan Plateau can improve the performance of lake simulation and facilitate the development of lake parameterization.
\end{abstract}

Keywords: Water temperature; thermal stratification; mixed layer depth; Tibetan Plateau; La'ang Co

湖泊作为地表的重要组成部分, 连接着大气圈和陆地水圈. 湖泊与大气间的能量和物质交换不仅影响

* 2020-04-30 收稿; 2020-07-09 收修改稿.

国家自然科学基金项目 $(41830650,91837208,91737205)$ 、第二次青藏高原综合科学考察研究项目 (2019QZKK0103)、中国科学院战略性先导科技专项 (XDA2006010103, XDA19070301) 和国家重点研发计划项目 (2018YFC1505701) 联合资助.

** 通信作者; E-mail:wqma@ itpcas.ac.cn. 
区域气候系统,也是环境变化的指示器 ${ }^{[1]}$. 湖泊的温度变化会引发热通量交换并改变湖表的风场,在湖气间 水热交换中起着关键作用 ${ }^{[2-4]}$. 湖泊的热结构也影响区域天气和气候系统 ${ }^{[5-6]}$, 同时影响流域内降水的分 布 ${ }^{[7]}$. 此外, 湖表温度直接响应大气环境的强迫, 进而影响湖泊内部热力层结的变化 ${ }^{[8]}$. 因此, 研究湖泊热力 分层变化特征,能加深我们对湖气间相互作用变化的认识, 有助于理解湖泊周边气候环境的变化规律.

湖泊热力结构不仅与外部环境相互作用,也驱动着水体内部的各种理化过程和动力过程. 水体受热膨 胀产生垂直密度差异, 进而形成稳定分层或发生混合. 湖泊最上层是湖泊混合层, 即温度、密度在垂直方向 分布基本均匀, 湍流旺盛的水体 ${ }^{[9]}$. 该层直接与大气进行物质和能量交换, 对水体整体的垂直输送具有重要 作用. 混合层下是温度梯度较大的温跃层和位于湖泊底部受扰动较少的湖下层, 温跃层较为稳定的层结结 构抑制了湖泊上下物质能量交换, 因此湖下层水体贫氧, 有大量营养物和污染物堆积 ${ }^{[10-11]}$. 当混合层深度较 小时, 水体呈较为稳定的热力层结, 将限制水中溶解物质的传递, 影响水中生物的养分供给 ${ }^{[12-14]}$. 因此, 湖泊 热分层与混合过程对水体中的溶解气体、营养物、污染物的垂直分布和输送、湖泊环境和水生生物群落变化 都有重要影响 ${ }^{[15-17]}$. 研究湖泊分层和混合层深度特征, 明确真实的湖水混合过程, 有利于深人理解湖泊内部 循环过程,了解水生环境状况,为水质问题做出预警.

青藏高原拥有地球上海拔最高、数量最多、面积最大的高原湖泊群, 湖泊数量和面积分别占全国总量的 $39.2 \%$ 和 $51.4 \%{ }^{[18]}$. 近年来, 受气候变化的影响, 长期缓慢的气温上升和短期极端的高温使湖泊的热力混合 过程发生了显著的变化 ${ }^{[19]}$. 其他地区的相关湖泊研究表明,气温升高会使热分层持续时间延长 ${ }^{[20]}$, 混合减 弱使湖泊底层缺氧 ${ }^{[21]}$, 秋季湖泊混合消失 ${ }^{[22]}$, 湖泊混合类型转变 ${ }^{[23]}$. 而青藏高原作为 “亚洲水塔” ${ }^{[24]}$, 升温 速率是全球温度变化的 3 倍左右 ${ }^{[25]}$, 而大多数高原湖泊的混合类型尚不明确. 青藏高原大部分湖泊都在经 历快速的扩张, 部分湖泊冰期缩短, 湖表温度升高, 分层时间延长 ${ }^{[26-28]}$. 但直于自然条件等因素的限制, 高原 湖泊的观测研究较少, 本就不明确的高原湖泊内部混合过程对气候变化的响应更容易被忽视. 因此, 明确当 前高原不同湖泊的混合类型和混合规律, 有利于明确高原湖泊类型和湖沼学特征, 了解高原湖泊生态的状 况,为高原湖泊模拟和变化评估提供参考.

目前国内水体热力分层和混合的研究面临的最主要问题就是观测资料的贯乏, 对于自然条件恶劣的高 原湖泊来说更是如此. 以往的研究虽然发展了一些利用常规气象资料和湖泊性质资料 (深度、透明度等) 模 拟湖泊温度的垂直分布的湖泊模型, 但在实际应用中仍需要水温的廓线观测进行校正 ${ }^{[29-30]}$. 例如 Flake 模型 模拟的湖表温度偏高, 热力层结发展快 ${ }^{[31]}$; CLM 模型模拟的表层温度偏低, 湖水混合不充分 ${ }^{[32]}$; GLM 模型 在夏季湖水分层时模拟的湖泊温度误差较大, 温跃层深度大于观测值 ${ }^{[33]}$. 而湖泊模式要求数据的时间分辨 率最好在 $1 \mathrm{~h}$ 以内 ${ }^{[34]}$, 高分辨率观测数据的贵乏使湖泊内部热力状况难以验证以及模式驱动数据存在误 差 ${ }^{[35]}$. 因此, 展现高原湖泊真实、连续的热力变化规律, 明确真实的混合过程, 加深对高原不同湖泊动力过 程和热力过程的理解,这也是本文的意义所在.

拉昂错作为藏南水系中面积较大的湖泊 ${ }^{[36]}$, 受西风和季风交替影响,对西风和印度季风引发的降水变 化非常敏感. 本研究基于高时间分辨率、连续的水体垂直温度变化数据, 对拉昂错的热力分层规律和混合层 深度变化进行探究, 并分析其影响因素, 旨在丰富高原湖泊热过程观测资料, 为进一步优化湖水混合参数 化,提高湖泊模拟能力提供参考.

\section{1 研究区域与方法}

\section{1 湖泊概况}

拉昂错 $\left(30^{\circ} 35^{\prime} \sim 30^{\circ} 51^{\prime} \mathrm{N}, 81^{\circ} 06^{\prime} \sim 81^{\circ} 19^{\prime} \mathrm{E}, 4572 \mathrm{~m}\right.$ a.s.l. $)$, 湖区位于我国西藏自治区普兰县, 属藏南山 地灌丛草原半干旱气候, 年均气温约 $2.0^{\circ} \mathrm{C}$, 年降水量 $150 \sim 200 \mathrm{~mm}^{[36-37]}$. 湖泊呈汤勺状, 北部为河流冲积扇, 深度较浅, 深度 $10 \mathrm{~m}$ 左右, 中间部分为狭长连接处, 深度 $25 \mathrm{~m}$ 左右, 南部为开阔湖区, 深度为 $40 \mathrm{~m}$ 左右, 最 大实测深度 $49.03 \mathrm{~m}^{[38]}$. 湖泊面积 $242.9 \mathrm{~km}^{2}$, 呈退缩趋势, 湖中露出多个岛屿, 长 $29 \mathrm{~km}$, 宽 $17 \mathrm{~km}$, 平均宽 $9.26 \mathrm{~km}^{[39]}$. 集水面积 $2551.5 \mathrm{~km}^{2}$, 主要依赖北部干噶河、那曲补给. 湖水 $\mathrm{pH}$ 值 8.6 , 属中度碳酸盐型微咸 水湖.

\section{2 观测方法}

湖水温度观测时段为 2019 年 6 月 3 日 0 时至 10 月 28 日 0 时, 时间间隔为 $15 \mathrm{~min}$. A 点 $\left(30.62^{\circ} \mathrm{N}\right.$, 
$81.18^{\circ} \mathrm{E}$ ) 为垂直温度梯度测点 (图 1), 水深 $39.5 \mathrm{~m}$. 观测仪器为美国 Onset 公司 U22 水温传感器, 温度计深 度分别为 $0.5 、 1 、 1.5 、 4.5 、 9.5 、 14.5 、 19.5 、 24.5 、 29.5 、 34.5$ 和 $39.5 \mathrm{~m} . \mathrm{B}$ 点 $\left(30.61^{\circ} \mathrm{N}, 81.17^{\circ} \mathrm{E}\right)$ 和 $\mathrm{C}$ 点 $\left(30.60^{\circ}\right.$ $\mathrm{N}, 81.26^{\circ} \mathrm{E}$ ) 为水面温度测点 (图 1), 处于湖岸边缘, 观测仪器为美国 Onset 公司 U20 可记录温度的水位传 感器. 由于西藏特殊的气候和地理环境, 拉昂错冰期较长, 冬季湖泊会被冰层完全覆盖. 湖泊结冰会将浮球 冻结在内, 冰层移动会带动仪器移动, 使测点发生偏移, 甚至可能使浮球因挤压而破裂, 造成仪器损坏或丢 失. 为保证观测数据的获取和可靠性, 设定观测时间为夏季和秋季 (6-10月).

气象观测的观测时段为 2019 年 6 月 6 日 0 时至 10 月 31 日 0 时,时间间隔 $30 \mathrm{~min}$. 自动气象站 $(\mathrm{AWS}$, Auto Weather Station, $30.63^{\circ} \mathrm{N}, 81.18^{\circ} \mathrm{E}$ ) 位于湖区南部湖心岛上(图 1). 观测仪器为英国 Gill 公司 GMX600、 SN-500 传感器及 DL1000 数据采集器组成的自动气象站, 在线计算半小时平均温度、气压、风速、湿度和四分 量辐射和半小时累计降水量, 采样频率 $10 \mathrm{~Hz}$. 同时选取中国气象局阿里地区普兰县气象站 $($ AWS-PL, 31.13 $\left.\mathrm{N}, 81.26^{\circ} \mathrm{E}\right)$ 气象数据与拉昂错湖区的气象观测进行比较和补充.

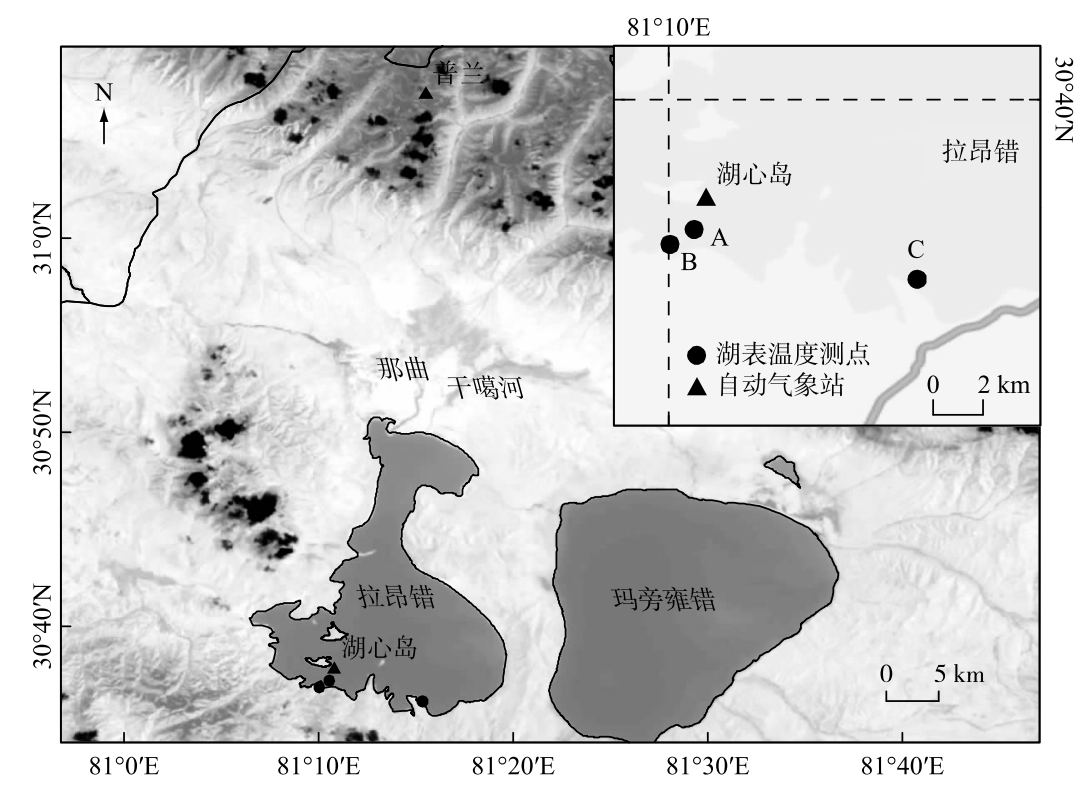

图 1 拉昂错湖温测站和自动气象站分布

Fig.1 Location of lake temperature observation sites and weather stations of La'ang Co

\section{3 混合层深度的计算}

混合层是水体表层温度、密度在垂直方向上分布基本均匀的层结, 混合层深度的确定对量化湖体层结 变化至关重要. 当混合层深度越大, 水体越偏向混合状态; 当混合层深度越小, 则说明混合作用较弱, 水体偏 向于层结状态. 依据 Kara 等 ${ }^{[9]}$ 的最佳标准 $\Delta T$, 根据混合层温度变化基本均匀为特点逐层寻找混合区域. 将 表层温度 $T_{1}$ 设为初始参考温度 $T_{\text {ref }}$, 根据上下两层的温差是否超出 $0.1 \Delta T$ 作为标准来确定水层是否均匀. 若 没有上下两层水体温差超过 $0.1 \Delta T$, 则说明水体温度在垂直方向上分布较为均匀, 水体完全混合, 此时混合 层深度为湖水深度; 若上下两层水体温差超过 $0.1 \Delta T$, 此时, $T_{\text {ref }}$ 为该层温度, 混合层底部温度 $T_{\mathrm{b}}$ 即为与当前 $T_{\text {ref 温度相差 }} \Delta T$ 的温度. 然后根据上下两层的温度与深度进行插值 ${ }^{[40]}$, 以减少廓线层数对混合层深度计算 的影响, 反算出更为合理的混合层深度. 具体方法如图 2 所示. 根据太湖和洱海的研究 ${ }^{[41]}$, 将参考标准 $\Delta T$ 设为 $0.5^{\circ} \mathrm{C}$,利用 MATLAB 编程进行计算.

\section{2 结果与分析}

\section{1 湖泊温度变化}

对观测期间湖泊剖面温度进行日平均, 得到拉昂错湖泊温度变化序列 (图 3). 6 月至 7 月中旬, 为水体 


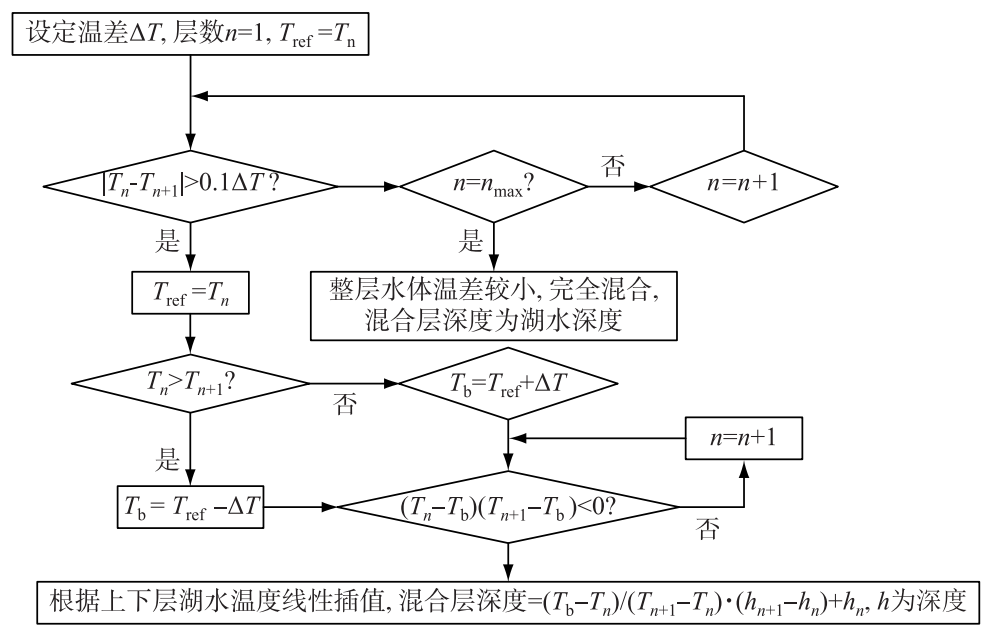

图 2 混合层深度计算流程

Fig.2 The mixed layer depth calculation process

的混合期, 整体水温较为均匀, 且水温逐渐升高, 表层水温从 $4.8^{\circ} \mathrm{C}$ 升至 $7.4^{\circ} \mathrm{C}$. 表层水和底层水之间偶尔出 现轻微的温度差, 但小于 $1^{\circ} \mathrm{C}$, 并不能阻止湖水的混合. 从 7 月中旬开始, 水体分层逐渐形成. 7 月下旬, 强烈 的湖面辐射加热和较低的风速促进了热力分层发展. 8 月开始, 表层水体温度迅速升高, 中下层水体相对升 温较慢, 水体层结结构逐渐明晰, 但 8 月中旬湖水分层消退, 可能由于风速增加使水体垂直湍流作用加剧, 导致湖水混合消解. 而后随着夏季太阳辐射的增强, 促进了水体热分层的发展, 湖面的快速变暖也会削弱对 流, 使热分层结构更加稳定 ${ }^{[42]}$. 整个 8 月份湖表和湖底平均温差为 $1.4^{\circ} \mathrm{C}$, 最大温差为 $3.2^{\circ} \mathrm{C} .9$ 月份太阳辐 射减弱, 表层湖水温度持续波动, 底层温度缓慢增高, 水表面和底部之间温度梯度减少, 湖水开始混合, 出现 上下翻转, 上层和底层温度趋于一致. 10 月由于气温降低, 水体不断向外释放热量, 表层水温由 $9.6^{\circ} \mathrm{C}$ 降至 $7.0^{\circ} \mathrm{C}$, 表层和底层平均温差 $0.3^{\circ} \mathrm{C}$. 从日平均湖水温度的季节变化来看, 拉昂错春季和秋季混合, 冬季结冰, 夏季有持续数日的稳定温度层结, 属于冷多次完全混合型湖泊. 和其他区域的湖泊相比, 青藏高原湖泊垂直 温度梯度变化较小 ${ }^{[43-46]}$, 可能与高原海拔较高, 空气温度较低有关.

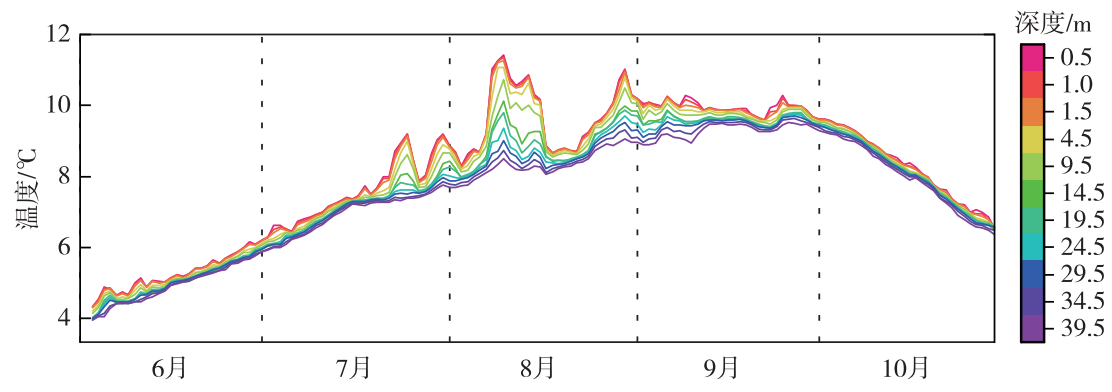

图 3 拉昂错日平均湖温季节变化

Fig.3 Seasonal variations of the average daily lake temperature in La'ang Co

从月平均日变化来看 (图 4), 拉昂错在下午 4 时左右湖水温度梯度最大, 白天垂直水温梯度较大, 夜晚 水温梯度较小. 白天由于太阳辐射的加热作用, 表层升温迅速, 湖水底层由于热传递的指数衰减而无法获得 热量, 易于出现温度层结, 而夜晚向外散失热量易于混合. 从图中也可以看出, 表层温度日变化较大, 底层温 度日变化较小. 夏季湖水分层期存在一日内持续性的温度层结, 但随着夏季温度升高, 整体水温也在上升, 夜晚湖泊向外辐射冷却也加剧了湖水的混合, 使水体以整层一致的趋势变化, 分层期较短, 这与拉昂错相较 
于其他高原湖泊更为宽浅有关.
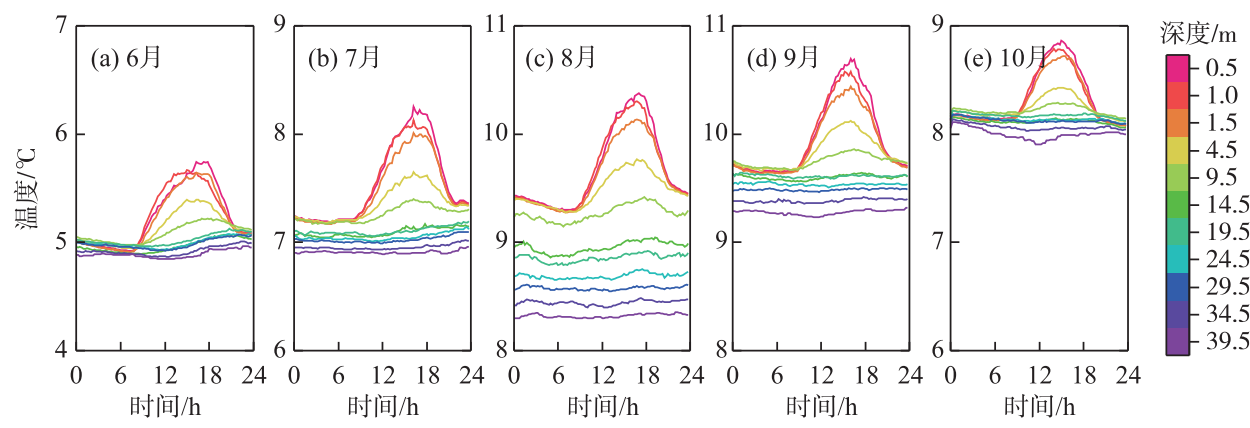

图 4 拉昂错月平均湖温日变化

Fig.4 Diurnal variations of the average monthly lake temperature in La'ang Co

研究表明, 区域空气温度和水表温度具有较好的一致性 ${ }^{[47-49]}$, 在湖泊研究中常利用空气温度或湖表温 度估算和预测湖泊热力学过程进而计算湖泊的能量收支 ${ }^{[50]}$. 由于拉昂错湖心岛自动气象站存在数据缺测 情况, 故利用中国气象局阿里地区普兰县气象站对空气温度数据进行补充. 从图 5 中可以看出, 普兰县自动 气象站观测的空气温度普遍高于湖心岛的空气温度, 对普兰县自动气象站观测的气温数据进行线性拟合, 其经验关系为 $y=-3.14+0.84 x$, 拟合后的均方根误差为 1.41 . 此外, 在目前的研究中, 由于观测站点的贵乏和 在湖中架设自动气象站的技术难度, 常利用湖泊周边气象站点的观测替代湖区的气象观测, 在没有湖中及 湖边邻近区域经风向篮除后的观测作为验证的情况下这种做法会带来较大的误差. 同时, 将拉昂错空气温 度与湖表温度 $(0.5 \mathrm{~m}$ ) 进行比较, 空气温度升温和降温更快, 变化幅度和湖表温度相比更为剧烈 (图 5 和图 6). 对日尺度来说, 观测期间空气温度最高为 $11.8^{\circ} \mathrm{C}$, 最低温度为 $-3.5^{\circ} \mathrm{C}$, 而水面温度最高为 $11.4^{\circ} \mathrm{C}$, 最低温 度为 $4.3^{\circ} \mathrm{C}$. 由于水的热容量较空气大, 水体升温和降温速率小于空气. 从图 5 可以看出, 气温峰值出现在 7 月底左右, 而湖中 $\mathrm{C}$ 点水表温度的峰值出现在 8 月底左右, 水表温度的峰值约滞后气温 1 个月, 且水体的降 温速率明显小于空气. 6 月和 7 月月平均气温高于水温, $8-10$ 月月平均水温高于气温. 对小时尺度来说, 空 气温度的日变化幅度同样高于水面温度 (图 6). 空气温度上午 8 时左右最小, 下午 4 时左右最大. 水面温度 上午 6 时左右最小, 下午 5 时左右最大, 日较差显著小于气温. 6 月开始气温逐渐升高, 7 月和 8 月气温日变 化大致相同, 9 月气温下降与 6 月大致相当, 10 月温度显著降低, 日最低温度低于 $0^{\circ} \mathrm{C}$. 而水面温度 $6-8$ 月 逐渐上升, 8 月和 9 月气温日变化大致相同, 10 月气温下降和 7 月大致相同. 湖表温度月均温度的高值出现 在 8 月和 9 月, 而空气月均温度的高值出现在 7 月和 8 月, 由此也可以看出水表温度变化滞后气温 1 个月 左右.

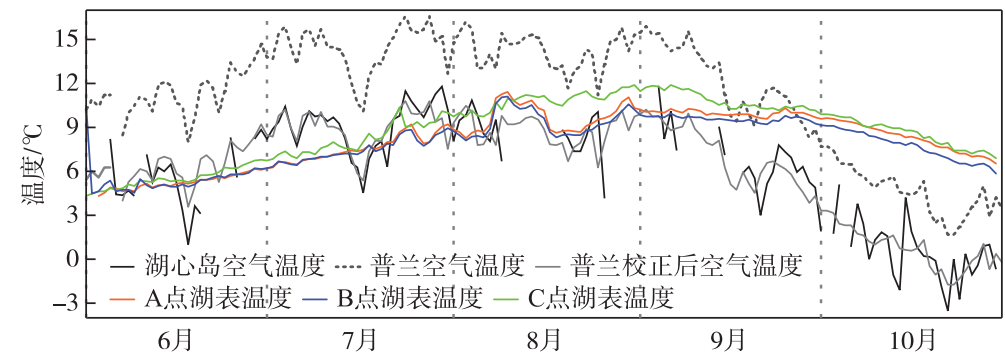

图 5 湖表温度和空气温度日平均月变化

Fig. 5 Monthly variations of lake surface temperature and air temperature

将湖面敞水区湖表温度和沿岸的湖表温度进行比较 (图 5), 拉昂错湖表温度在 3 个测点整体季节变化 较为一致,在湖泊分层期, 湖表温度存在较大的空间差异; 在混合期, 湖水混合较为均匀, 湖表温度的空间差 
异减小. 垂直梯度观测的 $\mathrm{A}$ 点和与 $\mathrm{A}$ 点距离较近的岸边 $\mathrm{B}$ 点温度变化基本一致, 从 6 月起温度逐渐上升, 8 月达到最大值, 9 月表层温度逐渐下降, 观测期间 $\mathrm{A}$ 点和 $\mathrm{B}$ 点的平均湖表温度分别为 8.2 和 $8.0^{\circ} \mathrm{C}$. 然而, $\mathrm{B}$ 点温度在秋季降温更快, 对空气温度的变化更为敏感. 距离较远的岸边 $\mathrm{C}$ 点与 $\mathrm{A}$ 点呈现出更大的空间差异, 6-9 月平均温度为 $8.8^{\circ} \mathrm{C}$, 比 $\mathrm{A}$ 点高出 $0.6^{\circ} \mathrm{C}$ 左右, 且 8 月温度较为平稳, 呈持续升温的态势, 没有出现与 $\mathrm{A}$ 点和 $\mathrm{B}$ 点相同的震荡. 因此, 利用岸线单点采集的表层水温数据估算湖泊整体的温跃层深度和湖泊储热量 会产生较大的误差.

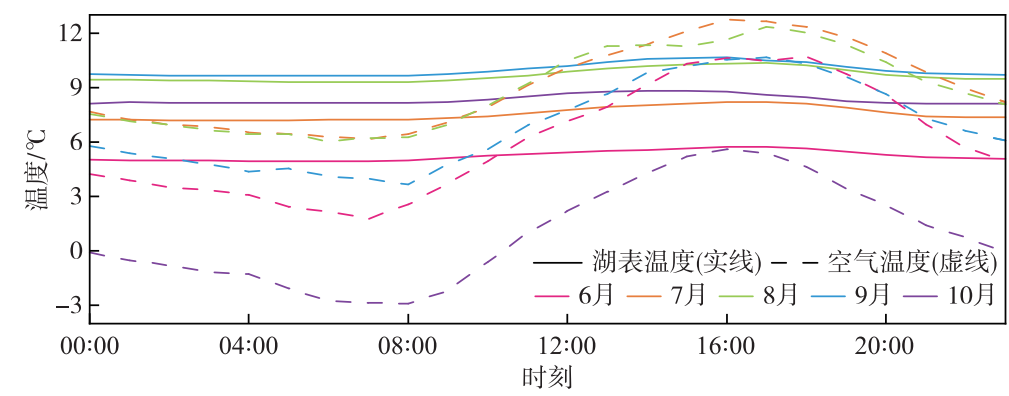

图 6 湖表温度和空气温度观测期间平均日变化

Fig.6 Diurnal variations of the average monthly lake surface temperature and air temperature

\section{2 水温垂直分布}

混合层是湖泊上部温度和密度混合相对均匀的层结, 其深度可以较为清晰的表征水体分层与混合过 程, 混合层深度越大说明湖水混合更为均匀. 利用观测资料对监测时段内湖泊混合层深度进行计算, 得到其 时间变化序列. 拉昂错的混合层深度在观测时段内总体上呈较为规律的日变化特征 (图 7), 夜间混合层深 度较大, 部分和湖水深度相同, 说明水体上下温度一致,整层湖水混合均匀; 白天混合层深度较小, 说明在混 合层深度处水体上下层存在较大的温度差异, 出现水体分层现象. 混合层深度的日变化特征与太阳辐射的 日变化特征较为吻合. 6 月至 7 月中旬, 混合层深度日变化特征较为规律, 夜间混合层深度为完整湖泊深度, 垂直温度一致, 充分混合, 白天表层混合层深度为 $10 \mathrm{~m}$ 左右, 湖水吸收太阳能使温度升高, 当风生流不足以 扰动整个水团,垂直温差增加. 7 月 15 和 16 日混合层深度维持为水体深度的时间增加, 出现了持续时间较 长的湖泊混合过程, 可能受云层覆盖的影响, 对应时间的太阳辐射异常偏小, 太阳辐射低于月均辐射, 而此 时风速高于 7 月平均风速, 较大风速引起的风力扰动施加于水体表面, 加速了湖泊的混合. 而后 17 日风速 突然下降, 有利于热力层结稳定, 对应湖泊混合层深度变小, 出现湖泊热力分层, 且持续时间较长. 7 月下旬 和 8 月中上旬, 混合层深度持续较小, 湖泊整体混合的持续时间缩短. 对应时刻太阳辐射较强, 风速较小, 有 利于热力层结的维持. 8 月 5 日太阳辐射高于月均辐射, 风速较小, 而后几天虽然辐射较小, 但风速也较小, 此时表层水体和深层水体的密度差异较大, 即使辐射减弱, 表层湖水也不会冷却到下沉的地步, 湖泊抵抗风 力和密度流扰动能力较强 ${ }^{[51]}$, 湖泊仍然可以维持持续性的温度层结结构. 8 月 7 日至 8 月 15 日, 混合层深 度持续较小, 湖泊处于持续性稳定分层期. 遗憾的是, 持续层结发生时对应时刻的气象数据缺测, 无法进行 更为准确的比较. 8 月 23 日及随后的几天风速较小, 辐射较强, 水体上层和下层温差逐渐增大, 能够有效阻 止水体混合, 随后又出现了持续多日的层结结构. 9 月上旬, 混合层深度呈现和 6 月大致相同的日变化. 而 9 月中旬开始, 分层时间缩短, 混合期增长. 尤其是 10 月 7 日和 8 日、19 日和 20 日,出现了持续性的湖水混合 过程. 结合气象数据, 这两段时间太阳辐射较小, 而风速较大, 使太阳辐射导致的微弱温差完全被风力混合, 湖泊向外释放热量. 因此, 太阳辐射和风速对热力层结的发展和消散起着重要的作用, 这与其他水体的研究 结果一致 ${ }^{[11]}$. 太阳辐射强时, 热力层结越稳定, 抑制水体垂直湍流交换, 混合层深度较小; 而风速大时, 动能 传人水体,引发混合过程,不利于热力层结的维持, 混合层深度较大.

\section{3 讨论}

湖泊是陆面过程研究的重要组成部分, 湖泊模型是研究湖泊过程和局地效应重要手段. 热力和风力作 


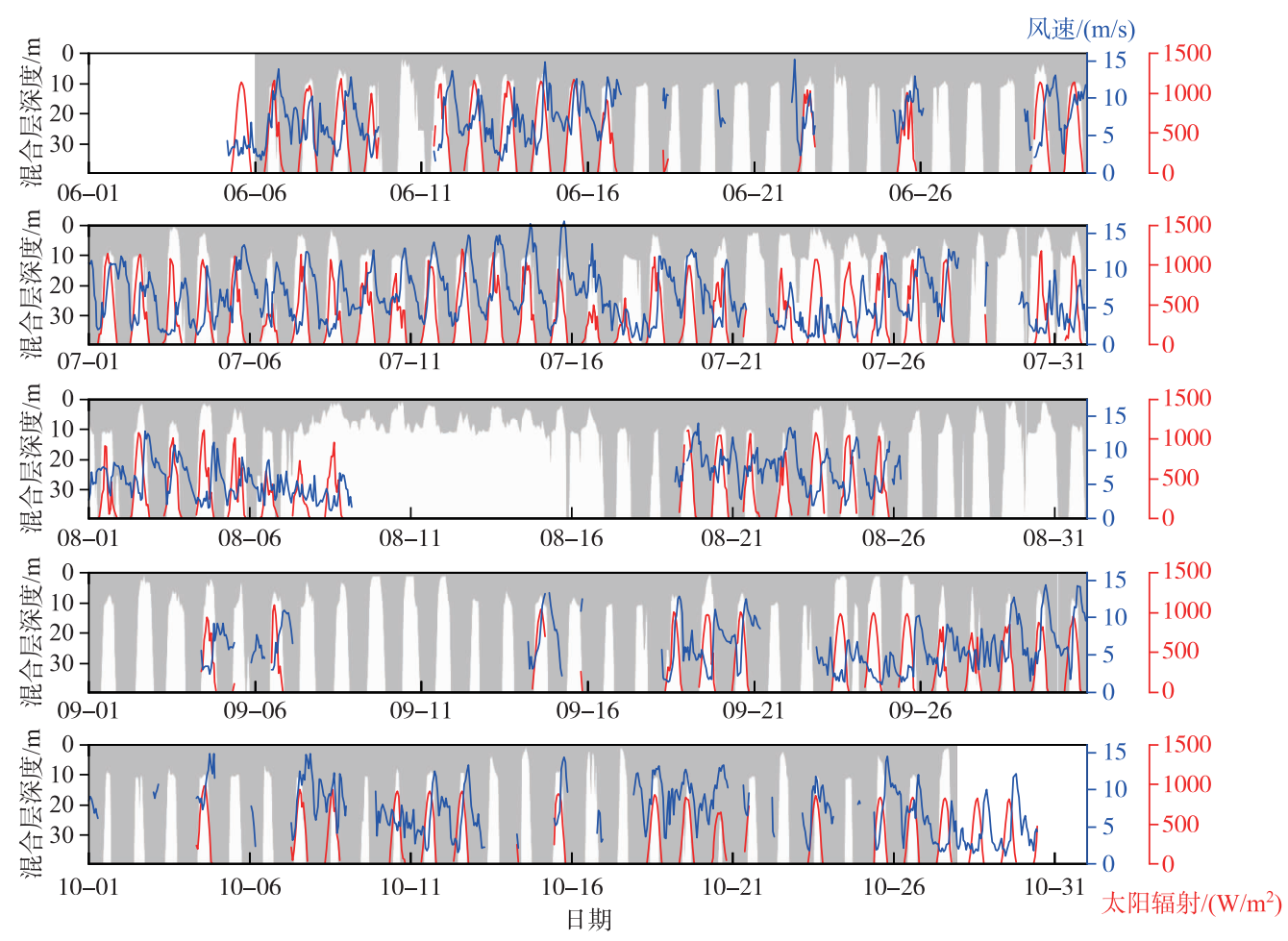

图 7 拉昂错混合层深度和气象要素的变化 (日期刻度线标记处为 $0: 00$ 时,灰色阴影为混合层深度)

Fig.7 The variations of mixed layer depth and meteorological elements of La'ang Co

(Abscissa ticks mark 0:00 AM, Grey shadow represents the depth of mixed layer)

为水体混合的主要外界驱动因子, 对湖泊的数值模拟至关重要. 对热力来说, 包含两个相反的作用 ${ }^{\left[{ }^{[52}\right]}$, 当水 体表层通过辐射作用获得热量时,表层水体升温比深层水体快,使水体密度随深度增加,形成分层; 而当表 层水温高于空气温度时, 水体中的热量向大气传输, 表层水体丧失热量, 使表层降温快于深层水体, 形成密 度梯度, 引发水体上下混合进而破坏原本稳定的水体层结结构. 对风力作用来说, 风力扰动使水体垂直湍流 作用加剧, 引发湖水混合, 消解湖水热力层结. 因此, 湖水的混合和分层状态主要受太阳辐射穿透和风力导 致的湍流混合共同作用,这也是现有湖泊模型都考虑的 2 个基本物理过程 ${ }^{[34]}$. Stepanenko 等指出目前的湖 泊模型对湖泊热过程模拟较差原因在于将水体混合过程简化为由近地表风速主导 ${ }^{[53]}$. Martynov 等指出强调 风生流主导的混合过程会弱化湖泊内部密度流对混合的作用, 使得春季湖表温度模拟出现不真实的增 暖 ${ }^{[54]}$. Maclntyre 等基于观测资料提出除风力作用外, 热力作用对热量混合同样起着至关重要的作用 ${ }^{[55]}$. 有 研究者通过提高湍流扩散系数来增强对湖泊混合过程的模拟 ${ }^{[56]}$, 但这种方法会高估湖底的混合过程 ${ }^{[57]}$. 因 此, 在利用湖泊模型对湖泊混合过程进行模拟时, 应当充分考虑热力和风力二者共同作用, 尤其对青藏高原 这种强辐射地区来说, 风速主导的混合方案会带来更大的误差.

根据太阳辐射和风力的强弱设定不同的阈值, 分别计算二者与混合层深度的相关系数 (表 1), 可以看出 太阳辐射和混合层深度的相关系数为负值, 风速和混合层深度的相关系数为正值, 辐射和风速对湖水分层 起相反的作用. 辐射越强, 混合层深度越小, 湖泊越容易分层; 风速越大, 混合层深度越大, 水体垂直混合更 为均匀. 当太阳辐射处于低值区时, 混合层深度与太阳辐射的相关性明显大于与风速的相关性, 此时处于夜 间或太阳升起下落时, 辐射变化较快, 表层水温升温和降温较为迅速, 辐射相比于风速对湖泊热过程的作用 更明显. 而当辐射处于高值区时, 二者与混合层深度的相关系数差异变小, 尤其是当辐射和风速都处于高值 区时. 此时往往处于下午辐射最强烈的时刻, 水体多处于分层期, 当风力作用很强时湖水才发生混合. 因此, 
高原湖泊湍流混合方案的建立应当充分考虑太阳辐射引起的暖层效应, 而不单单以风速为主建立参数化方 案, 以期使湖泊模拟更加准确.

表 1 不同条件下太阳辐射和风速分别与混合层深度的皮尔逊相关系数

Tab.1 The Pearson correlations of mixed layer depth with solar radiation and wind speed in different conditions

\begin{tabular}{|c|c|c|c|c|c|c|}
\hline \multirow{2}{*}{$\frac{\text { 太阳辐射 } /\left(\mathrm{W} / \mathrm{m}^{2}\right)}{\text { 风速 } /(\mathrm{m} / \mathrm{s})}$} & \multicolumn{2}{|c|}{$[0,400)$} & \multicolumn{2}{|c|}{$[400,800)$} & \multicolumn{2}{|c|}{$[800, \max ]$} \\
\hline & {$[0,10)$} & {$[10, \max ]$} & {$[0,10)$} & {$[10, \max ]$} & {$[0,10)$} & {$[10, \max ]$} \\
\hline 太阳辐射与混合层深度的相关系数 & $-0.36^{* *}$ & $-0.32^{* *}$ & $-0.30^{* *}$ & $-0.20 *$ & $-0.31^{* *}$ & $-0.29^{*}$ \\
\hline 风速与混合层深度的相关系数 & $0.10^{*}$ & 0.10 & $0.25^{* *}$ & 0.04 & $0.14^{*}$ & $0.26^{*}$ \\
\hline
\end{tabular}

**表示在 0.01 上显著相关, *表示在 0.05 上显著相关.

青藏高原已有的湖泊观测研究表明, 高原上大多数完全混合湖泊都属于双季对流型湖泊, 如班公错 ${ }^{[58]}$ 、 纳木错 ${ }^{[59]}$ 、佩枯错 ${ }^{[52]}$ 等. 这些湖泊一般冬季有冰层覆盖, 夏季稳定分层, 春季和秋季整层水体完全混合. 根 据 Lewis 湖泊类型分布图 ${ }^{[60]}$, 经海拔校正后青藏高原的大部分湖泊都应属于这种类型. 但是拉昂错的湖泊 观测资料显示, 该湖泊夏季稳定分层持续的时间很短, 仅数天, 分层不连续, 属于冷多次混合型湖泊, 不符合 Lewis 的经验分布. 这可能与拉昂错北浅南深的汤勺状的湖盆形状和冰川补给有关. 王君波等 ${ }^{[38]}{ }^{2010}$ 年 7 月对拉昂错湖温的单次测量也显示, 拉昂错湖水温度从表层至底层呈急剧递减趋势, 与东部的玛旁雍错相 比湖水温度变化单调, 未达到稳定分层, 可能与南部湖盆相对宽浅和补给不同有关. 因此, 高原地区湖泊混 合类型的确定存在很大的不确定性, 这也是缺乏观测验证的高原湖泊模拟不确定性的主要来源. 同时, 由于 8 月中旬同步气象观测资料的缺失, 无法判断两次分层中间间隔的混合期是否为气象因子的极端变化所导 致的异常情况, 需要长期的连续观测来确定. 此外, 湖泊的自身性质也会对湖水热力结构产生重要影响. 在 气候变化的背景下, 湖泊蒸发、降水、径流补给的变化会使湖泊的自身性质 (如面积、深度、盐度等) 发生变 化 ${ }^{[61]}$, 进而影响湖泊的混合过程. 而湖泊自身性质的变化在本研究中没有考虑在内, 如水体垂直盐度梯度 会影响垂直密度梯度进而影响水体垂直运动和混合过程, 需要结合观测在下一步研究中进行完善.

\section{4 结论}

本文通过分析青藏高原阿里地区拉昂错的湖泊温度和气象数据, 可以得出以下结论:

1) 拉昂错为冷多次完全混合型湖泊. 夏季间隔出现持续数天的分层期, 但不足以维持整个夏季. 春季和 秋季完全混合, 冬季结冰. 湖表温度 8 月达到最大值, 湖面敞水区和沿岸的湖表温度存在空间差异.

2) 与湖表温度相比, 拉昂错空气温度变化幅度更为剧烈, 降温更快. 6 月和 7 月月平均气温高于水温, 8-10月月平均水温高于气温, 湖表温度的峰值滞后气温约 1 个月.

3 ) 拉昂错混合层深度的日变化较为规律,下午混合层深度较小,存在水体分层现象,夜间混合层深度较 大, 水体垂直混合均匀. 太阳辐射和风是影响混合层深度变化的两个主要外界因子, 热力作用对拉昂错的湖 泊热过程作用更强.

致谢: 野外湖泊观测工作环境恶劣、条件艰苦, 本文作者衷心感谢中国科学院青藏高原研究所参与相关野外 工作的老师和同学们在湖上仪器架设和维护过程中的付出和帮助!

\section{5 参考文献}

[ 1 ] Bryan AM, Steiner AL, Posselt DJ. Regional modeling of surface-atmosphere interactions and their impact on Great Lakes hydroclimate. Journal of Geophysical Research: Atmospheres, 2015, 120(3) : 1044-1064. DOI: 10.1002/2014JD022316.

[ 2 ] Liu HZ, Feng JW, Sun JH et al. Eddy covariance measurements of water vapor and $\mathrm{CO}_{2}$ fluxes above the Erhai Lake. Scientia Sinica:Terrae, 2014, 44(11): 2527-2539. DOI: 10.1007/s11430-014-4828-1. [刘辉志, 冯健武, 孙绩华等. 洱 海湖气界面水汽和二氧化碳通量交换特征. 中国科学:地球科学, 2014, 44(11): 2527-2539.]

[ 3 ] Bonan GB. Sensitivity of a GCM simulation to inclusion of inland water surfaces. Journal of Climate, 1995, 8(11) : 2691 2704. DOI : 10.1175/1520-0442 (1995) 0082691 : soagst>2.0.co;2. 
[ 4 ] Lim S, Jang CJ, Oh IS et al. Climatology of the mixed layer depth in the East/Japan Sea. Journal of Marine Systems, 2012, 96/97: 1-14. DOI: 10.1016/j.jmarsys.2012.01.003.

[ 5 ] Liu AQ, Moore GWK. Lake-effect snowstorms over southern Ontario, Canada, and their associated synoptic-scale environment. Monthly Weather Review, 2004, 132(11) : 2595-2609. DOI: 10.1175/mwr2796.1.

[ 6 ] Swayne D, Lam D, MacKay M et al. Assessment of the interaction between the Canadian Regional Climate Model and lake thermal-hydrodynamic models. Environmental Modelling \& Software, 2005, 20(12) : 1505-1513. DOI: 10.1016/j.envsoft. 2004.08.015.

[ 7 ] Laird NF, Desrochers J, Payer M. Climatology of lake-effect precipitation events over lake Champlain. Journal of Applied Meteorology and Climatology, 2009, 48(2) : 232-250. DOI: 10.1175/2008jamc1923.1.

[ 8 ] Oswald CJ, Rouse WR. Thermal characteristics and energy balance of various-size Canadian shield lakes in the Mackenzie river basin. Journal of Hydrometeorology, 2004, 5(1) : 129-144. DOI: 10.1175/1525-7541 (2004) 0050129: tcaebo>2.0. co $; 2$.

[ 9 ] Kara AB, Rochford PA, Hurlburt HE. An optimal definition for ocean mixed layer depth. Journal of Geophysical Research: Oceans, 2000, 105( C7) : 16803-16821. DOI: 10.1029/2000JC900072.

[10] MacIntyre S, Flynn KM, Jellison R et al. Boundary mixing and nutrient fluxes in Mono Lake, California. Limnology and Oceanography, 1999, 44(3) : 512-529. DOI: 10.4319/lo.1999.44.3.0512.

[11] Abdulla CP, Alsaafani MA, Alraddadi TM et al. Mixed layer depth variability in the Red Sea. Ocean Science, 2018,14 (4) : 563-573. DOI: 10.5194/os-14-563-2018.

[12] Shimoda Y, Azim ME, Perhar G et al. Our current understanding of lake ecosystem response to climate change: What have we really learned from the north temperate deep lakes? Journal of Great Lakes Research, 2011, 37(1) : 173-193. DOI: 10. 1016/j.jglr.2010.10.004.

[13] Patterson JC, Hamblin PF, Imberger J. Classification and dynamic simulation of the vertical density structure of lakes. Limnology and Oceanography, 1984, 29(4) : 845-861. DOI: 10.4319/lo.1984.29.4.0845.

[14] Berger SA, Diehl S, Stibor H et al. Water temperature and mixing depth affect timing and magnitude of events during spring succession of the plankton. Oecologia, 2007, 150(4) : 643-654. DOI: 10.1007/s00442-006-0550-9.

[15] Chen DK, Busalacchi AJ, Rothstein LM. The roles of vertical mixing, solar radiation, and wind stress in a model simulation of the sea surface temperature seasonal cycle in the tropical Pacific Ocean. Journal of Geophysical Research: Oceans, 1994, 99(C10) : 20345-20359. DOI: 10.1029/94JC01621.

[16] Dodson SI. Introduction to limnology. Journal of the North American Benthological Society, 2004, 23(3): 661-662.

[17] Foley B, Jones ID, Maberly SC et al. Long-term changes in oxygen depletion in a small temperate lake: Effects of climate change and eutrophication. Freshwater Biology, 2012, 57(2) : 278-289. DOI : 10.1111/j.1365-2427.2011.02662.x.

[18] Ma RH, Yang GH, Duan HT et al. China's lakes at present: Number, area and spatial distribution. Scientia Sinica:Terrae, 2011, 41(3) : 394-401. [马荣华, 杨桂山, 段洪涛等. 中国湖泊的数量、面积与空间分布. 中国科学: 地球科 学, 2011, 41(3): 394-401.]

[19] Zhang YL. Effect of climate warming on lake thermal and dissolved oxygen stratifications: A review. Advances in Water Science, 2015, 26 (1) : 130-139. [张运林. 气候变暖对湖泊热力及溶解氧分层影响研究进展. 水科学进展, 2015, 26 (1) : 130-139.]

[20] Lee HW, Kim EJ, Park SS et al. Effects of climate change on the thermal structure of lakes in the Asian Monsoon Area. Climatic Change, 2012, 112(3/4) : 859-880. DOI: 10.1007/s10584-011-0233-3.

[21] Posch T, Köster O, Salcher MM et al. Harmful filamentous cyanobacteria favoured by reduced water turnover with lake warming. Nature Climate Change, 2012, 2(11) : 809-813. DOI: 10.1038/nclimate1581.

[22] Winder M, Hunter DA. Temporal organization of phytoplankton communities linked to physical forcing. Oecologia, 2008, 156( 1 ) : 179-192. DOI: $10.1007 / \mathrm{s} 00442-008-0964-7$.

[23] Peeters F, Livingstone DM, Goudsmit GH et al. Modeling 50 years of historical temperature profiles in a large central European lake. Limnology and Oceanography, 2002, 47(1) : 186-197. DOI: 10.4319/lo.2002.47.1.0186.

[24] Zheng D, Yao TD. Progress in research on formation and evolution of Tibetan Plateau with its environment and resource effects. China Basic Science, 2004, 6(2)：15-21. [郑度, 姚檀栋. 青藏高原形成演化及其环境资源效应研究进展. 中国基础科学, 2004, 6(2): 15-21.] 
[25] IPCC. Climate change 2014: Impacts, adaptation, and vulnerability. Part A: Global and sectoral aspects contribution of working group II to the Fifth assessment report of the intergovernmental panel on climate change. Cambridge: Cambridge University Press, 2014: 1132.

[26] Wang XW, Gong P, Zhao YY et al. Water-level changes in China's large lakes determined from ICESat/GLAS data. Remote Sensing of Environment, 2013, 132: 131-144. DOI: 10.1016/j.rse.2013.01.005.

[27] Song CQ, Huang B, Richards K et al. Accelerated lake expansion on the Tibetan Plateau in the 2000s: Induced by glacial melting or other processes? Water Resources Research, 2014, 50(4) : 3170-3186. DOI: 10.1002/2013wr014724.

[28] Zhu LP, Peng P, Zhang GQ et al. The role of Tibetan Plateau lakes in surface water cycle under global changes. J Lake $S c i, 2020,32(3)$ : 597-608. DOI : 10.18307/2020.0301. [ 朱立平, 彭萍, 张国庆等. 全球变化下青藏高原湖泊在地 表水循环中的作用. 湖泊科学, 2020, 32(3): 597-608.]

[29] Antonopoulos VZ, Gianniou SK. Simulation of water temperature and dissolved oxygen distribution in Lake Vegoritis, Greece. Ecological Modelling, 2003, 160(1/2) : 39-53. DOI: 10.1016/S0304-3800(02)00286-7.

[30] Momii K, Ito Y. Heat budget estimates for Lake Ikeda, Japan. Journal of Hydrology, 2008, 361(3/4) : 362-370.

[31] Thiery W, Martynov A, Darchambeau F et al. Understanding the performance of the FLake model over two African Great Lakes. Geoscientific Model Development, 2014, 7(1) : 317-337. DOI: 10.5194/gmd-7-317-2014.

[32] Zhang QH, Jin JM, Wang XC et al. Improving lake mixing process simulations in the Community Land Model by using $K$ profile parameterization. Hydrology and Earth System Sciences, 2019, 23 (12) : 4969-4982. DOI: 10.5194/hess-234969-2019.

[33 ] Huang L, Wang JB, Zhu LP et al. The warming of large lakes on the Tibetan Plateau: Evidence from a lake model simulation of nam co, China, during 1979-2012. Journal of Geophysical Research: Atmospheres, 2017, 122(24) : 13095-13107. DOI: $10.1002 / 2017$ JD027379.

[34] Ren XQ, Sun SF, Chen W et al. A review of researches on the lake numerical modeling. Advances in Earth Science, 2013, 28(3) : 347-356. [任晓倩, 孙菽芬, 陈文等. 湖泊数值模拟研究现状综述. 地球科学进展, 2013, 28(3): 347-356.]

[35] Su DS, Hu XQ, Wen LJ et al. Simulation of the response of Qinghai lake thermal conditions to climate change. Plateau Meteorology, 2018, 37(2): 394-405. [苏东生, 胡秀清, 文莉娟等. 青海湖热力状况对气候变化响应的数值研究. 高原气象, 2018, 37(2) : 394-405.]

[36] Qinghai-Tibet Plateau Comprehensive Scientific Expedition, Chinese Academy of Sciences ed. Rivers and lakes in Tibet. Beijing: Science Press, 1984. [中国科学院青藏高原综合科学考察队. 西藏河流与湖泊. 北京: 科学出版 社, 1984.]

[37] Wang SM, Dou HS ed. Chinese lakes. Beijing: Science Press, 1998: 406-409. [王苏民, 窦鸿身. 中国湖泊志. 北京: 科学出版社, 1998: 406-409.]

[38 ] Wang JB, Peng P, Ma QF et al. Investigation of water depth, water quality and modern sedimentation rate in Mapam Yumco and La'ang Co, Tibet. J Lake Sci, 2013, 25(4) : 609-616. DOI: 10.18307/2013.0420. [王君波, 彭萍, 马庆峰等. 西藏玛旁雍错和拉昂错水深、水质特征及现代沉积速率. 湖泊科学, 2013, 25(4) : 609-616.]

[39] La B, Bian D, Ci Z et al. Study on the change of lake area and its causes in the Mapangyong Co Basin in Tibet. Arid Zone Research, 2012, 29(6) : 992-996. DOI: 10.13866/j.azr.2012.06.028. [拉巴, 边多, 次珍等. 西藏玛旁雍错流域湖泊 面积变化及成因分析. 干旱区研究, 2012, 29(6): 992-996.]

[40] Yang YC, Wang YW, Zhang Z et al. Diurnal and seasonal variations of thermal stratification and vertical mixing in a shallow fresh water lake. Journal of Meteorological Research, 2018, 32(2) : 219-232. DOI: 10.1007/s13351-018-7099-5.

[41] Zhao QH, Sun JH. The variation features of the surface mixed layer depth in Erhai Lake and Taihu Lake in spring and autumn and their mechanism analyses? Acta Physica Sinica, 2013, 62(3) : 519-527. DOI: 10.7498/aps.62.039203. [ 赵巧 华, 孙绩华. 夏秋两季洱海、太湖表层混合层的深度变化特征及其机理分析. 物理学报, 2013, 62(3): 519-527.]

[42] Wetzel RG ed. Limnology: lake and river ecosystems. Gulf Professional Publishing, 2001.

[43] Livingstone DM. Impact of secular climate change on the thermal structure of a large temperate central European lake. Climatic Change, 2003, 57(1/2) : 205-225. DOI: 10.1023/a:1022119503144.

[44] Woolway RI, Merchant CJ. Worldwide alteration of lake mixing regimes in response to climate change. Nature Geoscience, 2019, 12(4) : 271-276. DOI: 10.1038/s41561-019-0322-x. 
[45] Zhang YL, Chen WM, Yang DT et al. Monitoring and analysis of thermodynamics in Tianmuhu Lake. Advances in Water Science, 2004, 15(1) : 61-67. [张运林, 陈伟民, 杨顶田等. 天目湖热力学状况的监测与分析. 水科学进展, 2004, 15(1): 61-67.]

[46] Bai Y, Zhang YL, Zhou YQ et al. Spatial distribution of the thermal stratification in Qiandao lake and its influencing factors. Oceanologia et Limnologia Sinica, 2016, 47(5) : 906-914. DOI: 10.11693/hyhz20160400095. [白杨, 张运林, 周 永强等. 千岛湖水温垂直分层的空间分布及其影响因素. 海洋与湖沼, 2016, 47(5) : 906-914.]

[47] Adrian R, O'Reilly CM, Zagarese H et al. Lakes as sentinels of climate change. Limnology and Oceanography, $2009, \mathbf{5 4}$ (6part2) : 2283-2297. DOI: 10.4319/lo.2009.54.6_part_2.2283.

[48] Zhang YX, Wang HM, Zhang R. Study on the relationship between surface temperature and air temperature of Fuxian lake. Environmental Science Survey, 2018, 37(4) : 26-29. [张月霞, 王慧梅, 张睿. 抚仙湖表层水温与气温关系研究. 环 境科学导刊, 2018, 37(4): 26-29.]

[49] Sun DM, Tian HF, Zhang H et al. Monitoring of water temperature and changing relationship between the water temperature and air temperature in the upper Yangtze River. Construction Conserves Energy, 2010, 38(12) : 74-77. [孙大明, 田 慧峰, 张欢等. 长江上游水温监测及水温和气温关系研究. 建筑节能, 2010, 38(12): 74-77.]

[50] Zhang YL, Wu ZX, Liu ML et al. Thermal structure and response to long-term climatic changes in Lake Qiandaohu, a deep subtropical reservoir in China. Limnology and Oceanography, 2014, 59(4) : 1193-1202. DOI: 10.4319/lo.2014.59. 4.1193.

[51] Kalff J ed. Limnology: inland water ecosystems. New Jersey: Prentice Hall, 2002.

[52] Lei Y, Yao T, Yang K et al. Thermal regime, energy budget and lake evaporation at Paiku Co, a deep alpine lake in the central Himalayas. Hydrology and Earth System Sciences Discussions, 2019, 421 : 1-27.

[53] Stepanenko VM, Goyette S, Martynov A et al. First steps of a lake model intercomparison Project: LakeMIP. Boreal Environment Research, 2010, 15:191-202.

[54] Martynov A, Sushama L, Laprise R. Simulation of temperate freezing lakes by one-dimensional lake models: performance assessment for interactive coupling with regional climate models. Boreal Environment Research, 2010, 15(2) :143-164.

[55] MacIntyre S, Fram JP, Kushner PJ et al. Climate-related variations in mixing dynamics in an Alaskan arctic lake. Limnology and Oceanography, 2009, 54(6part2) : 2401-2417. DOI: 10.4319/lo.2009.54.6_part_2.2401.

[56] Gu HP, Jin JM, Wu YH et al. Calibration and validation of lake surface temperature simulations with the coupled WRFlake model. Climatic Change, 2015, 129(3/4) : 471-483. DOI: 10.1007/s10584-013-0978-y.

[57] Subin ZM, Riley WJ, Mironov D. An improved lake model for climate simulations: Model structure, evaluation, and sensitivity analyses in CESM1. Journal of Advances in Modeling Earth Systems, 2012, 4 ( 1): M02001. DOI: 10. 1029/2011 MS000072.

[58] Wang MD, Hou JZ, Lei YB. Classification of Tibetan lakes based on variations in seasonal lake water temperature. Chinese Science Bulletin, 2014, 59(34) : 4847-4855. DOI: 10.1007/s11434-014-0588-8.

[59] Wang JB, Huang L, Ju JT et al. Spatial and temporal variations in water temperature in a high-altitude deep dimictic mountain lake ( Nam Co), central Tibetan Plateau. Journal of Great Lakes Research, 2019, 45(2) : 212-223. DOI: 10. 1016/j.jglr.2018.12.005.

[60] Lewis WM Jr. A revised classification of lakes based on mixing. Canadian Journal of Fisheries and Aquatic Sciences, 1983 , 40(10) : 1779-1787. DOI: 10.1139/f83-207.

[61] Zhu LP, Zhang GQ, Yang RM et al. Lake variations on Tibetan Plateau of recent 40 years and future changing tendency. Bulletin of Chinese Academy of Sciences, 2019, 34(11): 1254-1263. [ 朱立平, 张国庆, 杨瑞敏等. 青藏高原最近 40 年湖泊变化的主要表现与发展趋势. 中国科学院院刊, 2019, 34(11): 1254-1263.] 